N. Zamani, A. Keshavarz, M. Soliemanivareki / TJ MCS Vol .5 No. 2 (2012) 75-81

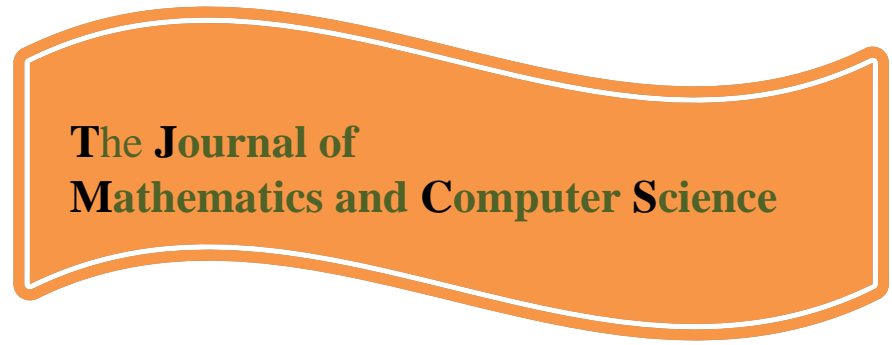

Available online at

http://www.TIMCS.com

The Journal of Mathematics and Computer Science Vol .5 No.2 (2012) 75-81

\title{
Optimization of nonlinear optical rectification coefficient in asymmetric double quantum wells
}

\author{
Naser Zamani ${ }^{1}$ \\ Department of Physics, College of Science, Shiraz University of Technology, Shiraz, Iran \\ n.zamani@sutech.ac.ir \\ Alireza Keshavarz \\ Department of Physics, College of Science, Shiraz University of Technology, Shiraz, Iran \\ keshavarz@sutech.ac.ir \\ Mohammad Soleimanivareki \\ Department of Applied Mathematics, Faculty of Basic Sciences, Islamic Azad University- Science Research \\ Ayatollah Amoli branch, Amol, Iran. \\ Mohammad_soleimanivareki@yahoo.com
}

Received: February 2012, Revised: November 2012

Online Publication: December 2012

\section{Abstract}

In this work, the particle swarm optimization is used as an optimization optical rectification coefficient for square double quantum wells. By combining this algorithm together with numerical solution of Schrödinger equation, and using the density-matrix method we found the wells structure that the optical rectification coefficient is maximum.

Application of this algorithm to the structure of asymmetric double quantum wells shows that the optical rectification coefficient is $6.82 \times 10^{-14} \mathrm{~V} / \mathrm{m}$.

Keywords: Particle Swarm Optimization; Optical Rectification Coefficient; Asymmetric Double Quantum Wells.

\section{Introduction.}

In the past two decades, much attention has been paid to the linear and nonlinear optical properties of semiconductor nanostructures such as quantum wells (QWs), quantum well wires (QWWs) and quantum dots (QDs) both theoretical and applied physics(Numai, 2004). There is currently a considerable interest in the optical phenomena based on intersubband transitions in

\footnotetext{
${ }^{1}$ Corresponding author. Faculty member Tel: +98 7117261392
} 
semiconductor quantum wells. Studies on these structures offer a wide range of potential applications for photo-electronic devices. Include semiconductor lasers, far-infrared laser amplifiers, infrared photo-detectors, optical memories, quantum computing, and all optical switches (Kristaedter, 1996; Faist et al., 1994; Choi et al., 1987).

In the present work, we optimize the nonlinear optical rectification associated with intersubband transitions within the conduction band for asymmetric double quantum wells (ASDQWs) which is composed of two quantum wells with different widths separated by a barrier. For this purpose, by the numerical methods we obtain the energy eigenvalues and eigenfunctions of the system and then using the particle swarm optimization method and by the variation of different values of the well parameters, width of barrier and mole fractional for two wells, obtain the optimum structure for rectification coefficient are determined.

The outline of the paper is as follows. In Section 2, with the compact density matrix approach and iterative method, the Hamiltonian, relevant eigenstates and eigenenergies, and the analytical expression for the linear and nonlinear susceptibility are described. In section 3 , mathematical model, the algorithm particle swarm optimizations are introduced and the rectification coefficient was applied function that we intent optimize the rectification coefficient. Our numerical results and a brief summary are presented in Sections 4 and 5, respectively.

\section{Theory.}

In the effective mass approximation, the one-dimensional Schrödinger equation with constant mass $\mathrm{m}^{*}$ of an electron confined in ASDQWs, is given by:

$$
\left(-\frac{\hbar^{2}}{2 m^{*}} \frac{d^{2}}{d z^{2}}+V(z)\right) \psi(z)=E \psi(z)
$$

where the one-dimensional V(z) is potential profile of the ASDQWs and introduced as:

$$
V(z)= \begin{cases}V_{0}, & z<-\left(L_{w 1}+\frac{L_{b}}{2}\right) \\ 0, & -\left(L_{w 1}+\frac{L_{b}}{2}\right)<z<-\left(\frac{L_{b}}{2}\right) \\ V_{0}, & -\left(\frac{L_{b}}{2}\right)<z<\left(\frac{L_{b}}{2}\right) \\ V_{1}, & \left(\frac{L_{b}}{2}\right)<z<\left(L_{w 2}+\frac{L_{b}}{2}\right) \\ V_{0}, & z>\left(L_{w 2}+\frac{L_{b}}{2}\right)\end{cases}
$$

The origin of the growth direction $\mathrm{z}$ is taken to be the center of the structure, $\psi(z)$ is the wave function. This structure consists of two GaAs well layers with widths $L_{w 1}$ and $L_{w 2}$, respectively, separated by an undoped $A l_{x} G a_{1-x} A s$ barrier layer with width $\mathrm{L}_{b}$. and also $\mathrm{V}_{0}=\mathrm{Q}_{c} \Delta \mathrm{E}_{g 1}$ and 
N. Zamani, A. Keshavarz, M. Soliemanivareki / TJ MCS Vol .5 No. 2 (2012) 75-81

$\mathrm{V}_{1}=\mathrm{Q}_{c} \Delta \mathrm{E}_{g 2} . \mathrm{Q}_{c}$ is band offset ratio and $\Delta \mathrm{E}_{g}$ is the band-edge discontinuities between a GaAs and $A l_{x} G a_{1-x}$ As an heterojunction.

By using the numerical method one can be obtained the energy levels and corresponding wave function for electrons in conduction band of ASDQWs. For calculation nonlinear optical rectification coefficient corresponding to the optical transitions between two energy eigenstates, it must be used the density-matrix method(Ahn and Chuang, 1987; Kelin et al. 1991).

Assume that the system under consideration is excited by an electromagnetic field such as:

$$
\begin{aligned}
E(t) & =E_{0} \cos \omega t \\
& =\tilde{E} e^{i \omega t}+\tilde{E} e^{-i \omega t},
\end{aligned}
$$

where $\omega$ is the external field-frequency applied to the system with a polarization vector normal to the ASDQWs along the $\mathrm{z}$-direction. Then, the time-dependent equation of the density matrix operator $\rho$ is given by:

$$
\frac{\partial \rho}{\partial t}=\frac{1}{i \hbar}\left[H_{0}-q z E(t), \rho\right]-\Gamma\left(\rho-\rho^{(0)}\right),
$$

where $H_{0}$ is the Hamiltonian for this system without the electromagnetic field $E(t), q$ is the electronic charge, $\rho^{(0)}$ is the unperturbed density matrix operator, [,] is the quantum mechanical commutator and $\Gamma$ is the operator responsible for the damping due to the electron-phonon interaction, collisions among electrons, etc. It is assumed that $\Gamma$ is a diagonal matrix and its elements are equal to the inverse of relaxation time T. Eq. (4) can be solved using an usual iterative method(Boyd, 2003):

$$
\rho(t)=\sum_{n} \rho^{(0)}(t)
$$

With

$$
\begin{aligned}
\frac{\partial \rho_{i j}^{(n+1)}}{\partial t} & =\frac{1}{i \hbar}\left\{\left[H_{0}, \rho^{(n+1)}\right]_{i j}-i \hbar \Gamma_{i j} \rho_{i j}^{(n+1)}\right\} \\
& -\frac{1}{i \hbar}\left[q z, \rho^{(n)}\right]_{i j} E(t) .
\end{aligned}
$$

The electronic polarization of the square quantum wells can be expanded as Eq.(5). We will restrict ourselves to considering the first two orders, i.e[8].

$$
P(t)=\varepsilon_{0}\left(\chi_{\omega}^{(1)} \tilde{E} e^{(i \omega t)}+\chi_{2 \omega}^{(2)} \tilde{E}^{2} e^{(2 i \omega t)}\right)+\text { c.c. }+\varepsilon_{0} \chi_{0}^{(2)} \tilde{E}^{2},
$$

where $\chi_{\omega}^{(1)}, \chi_{2 \omega}^{(2)}$ and $\chi_{0}^{(2)}$ denote the linear, second-harmonic generation, and optical rectification coefficient, respectively. $\varepsilon_{0}$ is the vacuum permittivity.

Using the same density matrix formalism as in(Boyd, 2003), we have calculated the expression of $\chi_{0}^{(2)}$ for this model(Rezaei et al. 2010):

$$
\chi_{0}^{(2)}=\frac{2 \sigma_{v}}{\varepsilon_{0} \hbar^{2}} \mu_{12}^{2}\left|\mu_{22}-\mu_{11}\right| \times \frac{E_{21}^{2}}{\left[\left(E_{21}-\hbar \omega\right)^{2}+\left(\hbar \Gamma_{12}\right)^{2}\right]\left[\left(E_{21}+\hbar \omega\right)^{2}+\left(\hbar \Gamma_{12}\right)^{2}\right]},
$$


N. Zamani, A. Keshavarz, M. Soliemanivareki / TJ MCS Vol .5 No. 2 (2012) 75-81

where $\sigma_{v}$ is the carrier density, $M_{i j}=\left|\left\langle\psi_{i}|q \hat{z}| \psi_{j}\right\rangle\right|(i, j=1,2)$ are the dipole matrix element, $E_{i j}=E_{i}-E_{j}$ is the energy interval of the two level system, and $\Gamma$ shows the damping due to electron-photon interaction. $\mu$ is the permeability of the system, $c$ is the speed of light in the free space and $I=2 \varepsilon_{0} n_{r} c|E|^{2}$ is the incident optical intensity of the electromagnetic field which $n_{r}$ is the refractive index.

\section{Optimization Model}

In this paper the PSO is used to optimize the ASDQWs structure in order to maximize the nonlinear optical rectification coefficient. PSO algorithm is a population-based stochastic optimization method. The algorithm is derived by the social behavior of bird flocks, colonies of insects, schools of fishes, and groups of animals feeding and traveling together. The algorithm is started by initializing a population of random solutions called particles and searches for the best position by updating production through the following velocity and position update equations. The velocity and position are updated by the following equations:

$$
\begin{aligned}
V_{j}^{k+1} & =\omega \times V_{j}^{k}+C_{1} \times \operatorname{rand}(\cdot)\left(P_{\text {Best }, j}^{k}-X_{j}^{k}\right)+C_{2} \times \operatorname{rand}(\cdot)\left(G_{\text {Best }, j}^{k}-X_{j}^{k}\right), \\
X_{j}^{k+1} & =X_{j}^{k}+V_{j}^{k+1},
\end{aligned}
$$

where $j=1,2, \ldots, N_{\text {Swarm }}$ is the index of each particle, $N_{\text {Swarm }}$ is the number of the swarms, $k$ is the current iteratin number, rand (.) is random numbers between 0 and $1 . V_{j}^{k}$ is the current velocity of particle $j$ at iteration $k, V_{j}^{k+1}$ is the modified velocity of particle $j$ at iteration $k+1, X_{j}{ }_{j}^{k}$ is the current position of particle $j$ at iteration $k, P_{B e s t, j}$ is the optimal coordinate value of the $k$ th particle obtained so far and $G_{\text {Best }}$ is the best coordinate value found so far in the whole swarm. Constants $C_{1}$ and $C_{2}$ are the weighting factors of the stochastic acceleration terms, which pull each particle towards the $P_{B e s t, j}$ and $G_{\text {Best }}$. $\omega$ is a non-negative constant called inertia weight and used to control the convergence behavior of the PSO(Kennedy, 1997).

\section{3-1. Application of the PSO for nonlinear optical rectification coefficient of ASDQWs}

The main steps of optimization are as follows:

Step1. Initialize stochastically positions of the particles in the pre-specified ranges.

Here the positions represent the width of two wells and barrier and mole fractional for two wells.

In practice, if boundaries exist the following restrictions also need to be enforced: $X_{j} \in\left[X_{\min }, X_{\max }\right]$, where $X_{\min }$ and $X_{\max }$ are pre-specified constants. Based on this, the PSO algorithm will be able to find the global optimum point in sequence iterations.

Step2. Usage of five input data for solving Schrödinger equation to calculate eigenenergy and eigenfunction and the calculate the absorption coefficient for initial populations. For each particle $j$, the velocity and position of particles can be updated. 
N. Zamani, A. Keshavarz, M. Soliemanivareki / TJ MCS Vol .5 No. 2 (2012) 75-81

Step3. Update the positions according to formula(9) and (10) and then go back to step 2.

Step4. Terminate the optimization process and print the result if the target of optimization is met, or the allowed maximum number of iteration is reached.

\section{Results and Discussion}

Optical properties of quantum wells are strongly related to the energy eigenvalues and their corresponding wave functions. Having given that a small change in the width of the wells, barrier and mole fractional of $\mathrm{Al}$ change the energy eigenstates and wave functions of the system. The parameters used in this paper are as follows:

$m_{\text {GaAs }}^{*}=0.067 m_{0}, \quad m_{A l G a A s}^{*}=(0.067+0.083 x) m_{0}$ where $m_{0}$ is the mass of the free electron, $\sigma_{v}=3 \times 10^{22} \mathrm{~m}^{-3}, n_{r}=3.2, T_{21}=0.2 p s$ where $\Gamma_{21}=1 / T_{21}, I=0.6 \times 10^{10} \mathrm{~W} / \mathrm{m}^{2}$ and $\Delta \mathrm{E}_{g}=1.247 \mathrm{x}$ ( $x$ corresponding mole fractional of $A l$ ). Using the above optimization algorithm we determine the optimal structure parameter. In order to use the PSO algorithm, minimum amounts which there are two energy levels for quantum wells are obtained. Rang of particles in the width and height of two wells and separated by a barrier are to be: $L_{w 1} \in[35,70] A^{\circ}, L_{w 2} \in[35,70] A^{\circ}$, $L_{b} \in[15,30] A^{\circ}, V_{0} \in[100,300](m e V)$ and $V_{1} \in[100,300]$ ( $\left.m e V\right)$ Results obtained using the PSO algorithm are shown in Table.1.

Table 1. Ruslts of PSO

\begin{tabular}{|c|c|c|c|c|}
\hline$L_{w 1}\left(A^{\circ}\right)$ & $L_{w 2}\left(A^{\circ}\right)$ & $L_{b}\left(A^{\circ}\right)$ & $V_{0}(m e V)$ & $V_{1}(m e V)$ \\
\hline 70 & 48.38 & 29.73 & 100 & 100.0016 \\
\hline
\end{tabular}

As the PSO algorithm is a random statistical algorithm, the typical convergence plots of PSO for finding optimal absorption for 100 iterations is shown in Fig.1. In this figure shows that by using the PSO algorithm after 40 iteration optical rectification coefficient has a constant value which is equal to $6.82 \times 10^{-14} \mathrm{~V} / \mathrm{m}$. The optimum structure of double quantum wells depicts in Fig.2. includes the first two wave functions of ASDQWs. Seen that optimum structure is asymmetric and using the numerical solutions, ground state energy and its first excited state of $E_{1}=35.743510227065(\mathrm{meV})$ and $E_{2}=53.415828019709(\mathrm{meV})$, respectively.

As respects the shape of double quantum well is asymmetric, variation of the optical rectification coefficient versus of the photon energy is plotted in Fig.3. This indicates that resonance peak at photon energy value of $17.672(\mathrm{meV})$ as expected corresponding to difference between two first energy levels. From this figure we see that the magnitude of the resonant peak is $6.82 \times 10^{-14} \mathrm{~V} / \mathrm{m}$. 
N. Zamani, A. Keshavarz, M. Soliemanivareki / TJ MCS Vol .5 No. 2 (2012) 75-81

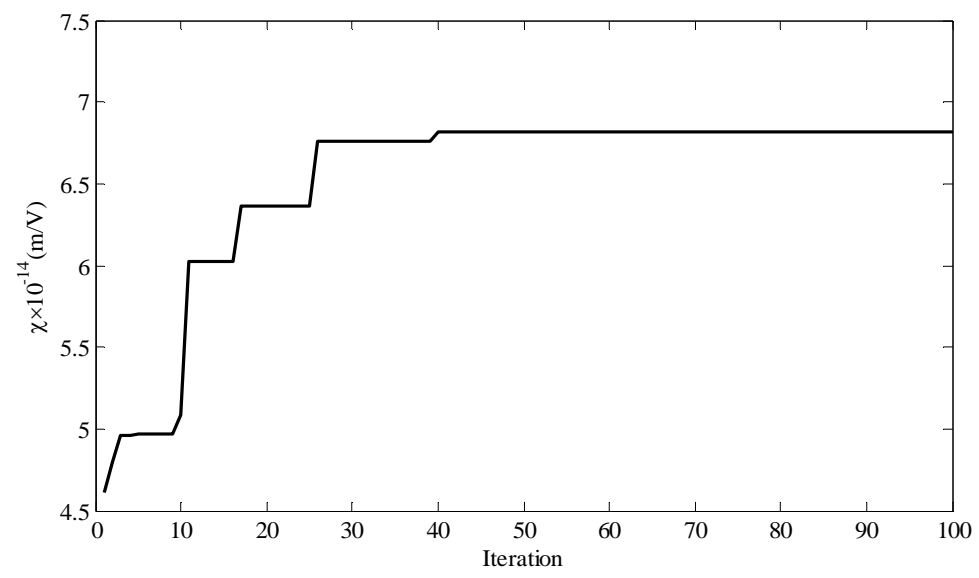

Figure 2. Convergence plot of PSO for finding optimal rectification.

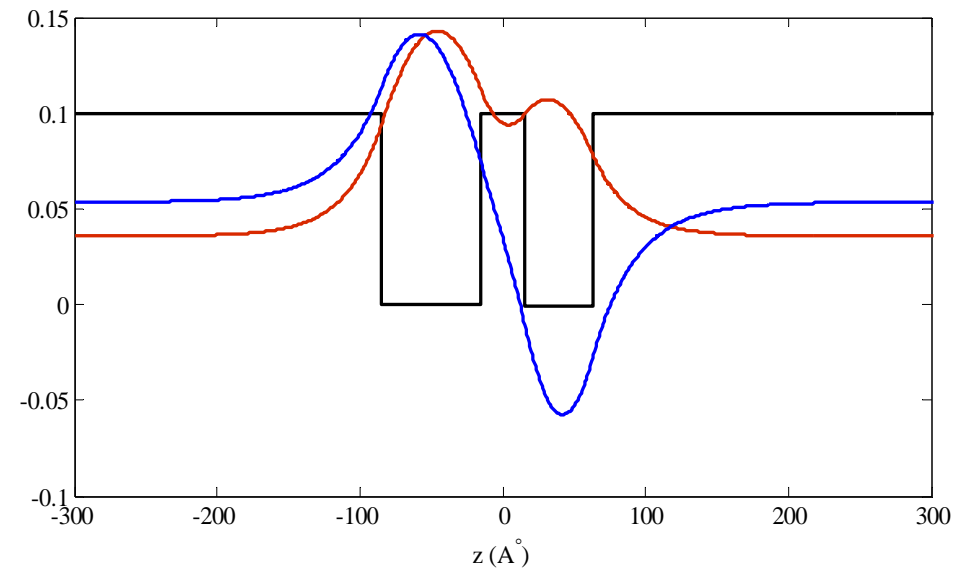

Figure 1. Schematic diagram for an asymmetric double quantum well for optimal parameters with first and second wave function.

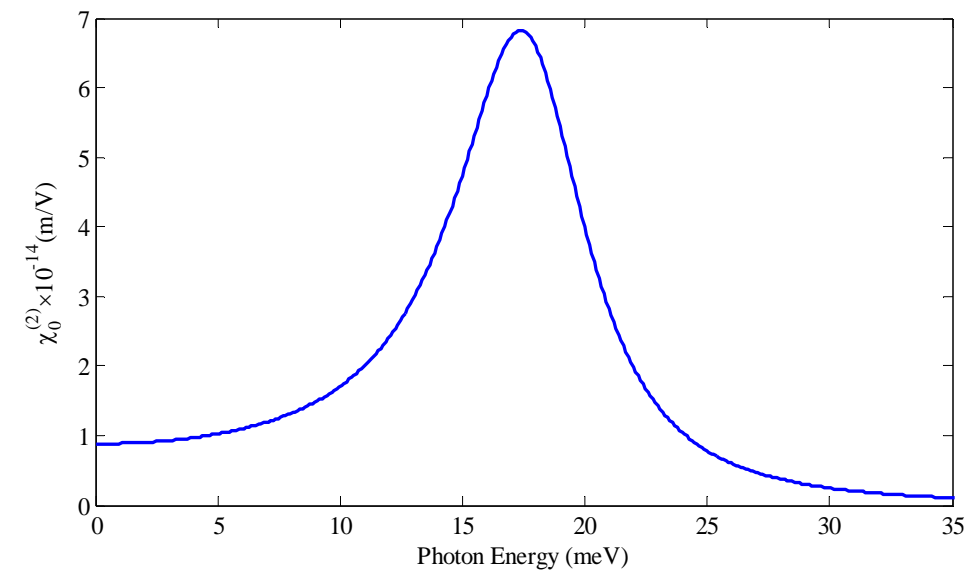

Figure 3. Variations of optical rectification coefficient versus the photon energy. 


\section{Conclusion}

In conclusion, we have performed the numerical calculations to solve the Schrödinger equation in order to find energy eigenvalues and eigenfunctions of ASDQWs. The calculations mainly focus we to find the optimum parameters to maximize the nonlinear optical rectification coefficient. For this purpose we used the particle swarm optimization.

Acknowledgment. The authors would like to thank the Islamic Azad university-science research Ayatollah Amoli branch for support this research.

\section{References.}

[5] D. Ahn and S. L. Chuang, Intersubband optical absorption in a quantum well with an applied electric field, Phys. Rev. B., 35 (1987), 4149-4151.

[8] R. W. Boyd, "Nonlinear Optics", 2nd ed., Academic Press, 2003.

[4] K. K. Choi, B. F. Levine, C. G. Bethea, J. Walker, R. J. Malik, Multiple quantum well $10 \mu \mathrm{m} \mathrm{GaAs}$ /AlxGa1-xAs infrared detector with improved responsivity, Appl. Phys. Lett, 50 (1987), 1814-1817. [3] J. Faist, F. Capasso, D. L. Sivco, C. Sirtori, A. L. Hutchinson, A.Y. Cho,Quantum cascade laser, Science, 264 (1994), 553-556.

[6] J. Kelin, Kuhn Gita, U. Lyengar, Sinclair. Yee, Free carrier induced changes in the absorption and refractive index for intersubband optical transitions in AlxGa1-xAs/GaAs/AlxGa1-xAs quantum wells, J. Appl. Phys 70 (1991), 5010-5018.

[10] J. Kennedy, The particle swarm: social adaptation of knowledge, IEEE Int. Conf. Evol. Comput. 1997.

[2] N. Kristaedter, Gain and differential gain of single layer InAs/GaAs quantum dot injection lasers, Appl. Phys. Lett. 69 (1996), 1226-1228.

[1] T. Numai, "Fundamentals of Semiconductor Lasers", Springer, New York, 2004.

[9] G. Rezaei, M. J. Karimi, A. Keshavarz, Excitonic effects on the nonlinear intersubband optical properties of a semi-parabolic one-dimensional quantum dot, Physica E, 43 (2010), 475-481.

[7] S. Unlu, I. Karabulut, H. Safak, Linear and nonlinear intersubband optical absorption coefficients and refractive index changes in a quantum box with finite confining potential, Physica E, 33 (2006), 319-324. 